\title{
Some aspects and aerodynamic effects in repairing battle damaged wings
}

\author{
S. Djellal ${ }^{1} \&$ A. Ouibrahim ${ }^{2}$ \\ ${ }^{I}$ Fluid Mechanics Laboratory, EMP, Bordj El Bahri, Algiers, Algeria \\ ${ }^{2}$ Laboratoire Energétique, Mécanique et Matériaux, \\ Université de Tizi-Ouzou, Algeria
}

\begin{abstract}
This paper concerns an experimental investigation, conducted to estimate the aerodynamic effects of aircraft battle-damage repair patches. Of particular concern in this study are the wings. Three different repair schemes are considered, lower (repair at the intrados), upper (repair at the extrados) and full repairs (both intrados and extrados at the same time). Preliminary results clearly showed that repair patches improve the aerodynamic performances of the damaged aircraft model. The full repair was shown to achieve the best recovery of the aerodynamic performances provided that the patch is thin enough. Repairs performed on only one surface (i.e. upper or lower) were also shown to provide significant aerodynamic improvements. The improvement tendency decreases with the increase of the patch thickness. Above a certain thickness value, the fineness decreases below the fineness of the damaged unrepaired model.
\end{abstract}

Keywords: aerodynamic performance, battle-damage, fineness, patch, repair.

\section{Introduction}

Military airplanes may return from battle flights with some form of battle damage affecting their aerodynamic performances. Moreover, damage may also occur even on the ground, between two operations. This damage should be treated as battle damage in operational scenarios regardless of its origin. To ensure availability, airplanes must be restored as quickly as possible. This will be accomplished through procedures and repair techniques that are substantially different from those used in time of peace. Successful repairs of battle damage 
result from quick and accurate assessment, coupled with simple and efficient repair techniques.

In a similar way we have to point out the interest in airplane safety which arose after the Aloha accident $[1,2]$ for civil aviation and after the Royal Australian Air force (RAAF) Machi accident [2] for military aviation associated to the presence of the cracks [3]. If the number of crack is limited and the crack or hole size is small compared to the damaged component, repairs are less expensive, otherwise the component should be replaced [4].

Possible repairs include either replacement of a fastener or filling or damage removal or patching. Metallic reinforcement with rivets and bolts [5] is the traditional method for repairing airplane cracked structures. The use of metallic sheets involves problems such as fatigue, galvanic corrosion, and difficult inspection after repair [6].

Baker [2] has been a pioneer in the design and assessment of repairs in 1970 at the Australian Research Laboratory (ARL) where he worked for the RAAF. Among his colleagues, Jones introduced the adhesively bonded repairs in the military aviation.

Unfortunately, very little work has been undertaken to study the aerodynamic effects of repairs devoted to the case of two-dimensional wings [7].

The objective of this investigation is to obtain estimates of the aerodynamic effects of aircraft battle-damage repair (ABDR) patches for an aircraft model. The investigation concerns the influence of different repair schemes and thicknesses.

\section{Repair modelling}

The effect of repair on the performance of the model is investigated with a battle context in mind, where in some combat situations it may be appropriate to carry out rapid temporary repairs. Such repairs may consist of simple patches installed over the battle-damaged area to cover perforations in the skin. Hence, three repair schemes are considered in our study:

Extrados (upper surface) repair with no lower surface repair.

Intrados (lower surface) repair with no upper surface repair.

Full repair: repairs on both upper and lower surfaces of the wing.

The model of aircraft being used is the same as the one studied by Djellal [8] concerning the effect of the damage. In this study, we consider two damage holes of the same diameter (Table 1, representing $40 \%$ of the local chord). These holes are located at different spanwise wing positions, which are the root region and between the tip region and the m.a.c. (mean aerodynamic chord). These locations are labelled position 1 and 2 respectively in Fig. 1. In addition, they are both centred at mid-chord.

The magnitude of the hole diameter as well as the position 1 were selected in order to have maximum performance loss due to the damage [8], hence expecting a larger improvement upon repair. Position 2 is selected for comparison purposes. 
Repair patches are made of aluminium sheet and are circular in shape. It was shown in a previous study devoted to repaired two-dimensional wings that circular patch gives slightly more recovery in aerodynamic performance lost by damage than the square one [9]. Patches were attached to the surface of the wing with $0.2 \mathrm{~mm}$ sided sticking tape. Table 1 gives the damage diameters subjected to repairs as well as dimensions of the patches used for the two positions.

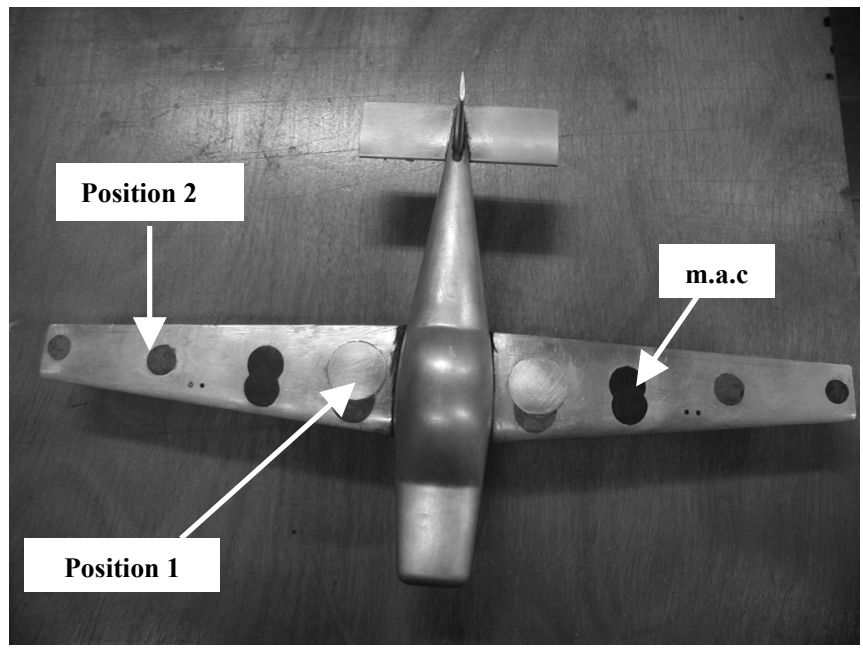

Figure 1: $\quad$ Model and repair patches.

Table 1: $\quad$ Damage and repair dimensions.

\begin{tabular}{lcc}
\hline \hline & Position 1 & Position 2 \\
\hline Damage diameter & $24.5 \mathrm{~mm}$ & $18.5 \mathrm{~mm}$ \\
Circular patch diameter & $35 \mathrm{~mm}$ & $25 \mathrm{~mm}$ \\
\hline
\end{tabular}

\section{Experimental program}

The experimental setup used (Fig. 2) has been presented in detail elsewhere [8] and is described here only briefly. All tests were conducted in the subsonic closed return wind tunnel at Polytechnic school of Algiers. This has a $0.6 \mathrm{~m}$ diameter test section and is capable of wind speed up to $48 \mathrm{~m} / \mathrm{s}$. For a velocity of $45 \mathrm{~m} / \mathrm{s}$, the Reynolds number used, based on the wing mean chord, was $1.5 \times 10^{5}$. Although this value is much smaller than the full scale cruise flight value $\left(3.9 \mathrm{X} 10^{6}\right)$, it is still not below the critical values and the understanding of the flow behaviour and repair influence can be explained. Force and moment were measured on a three-component balance (Fig. 2). The accuracy measurement of each component is $0.05 \%$. The acquisition system gives repeatability of the lift coefficient $\mathrm{C}_{\mathrm{L}}$ to within $0.5 \%$, and the drag coefficient $\mathrm{C}_{\mathrm{D}}$ to $0.2 \%$. 


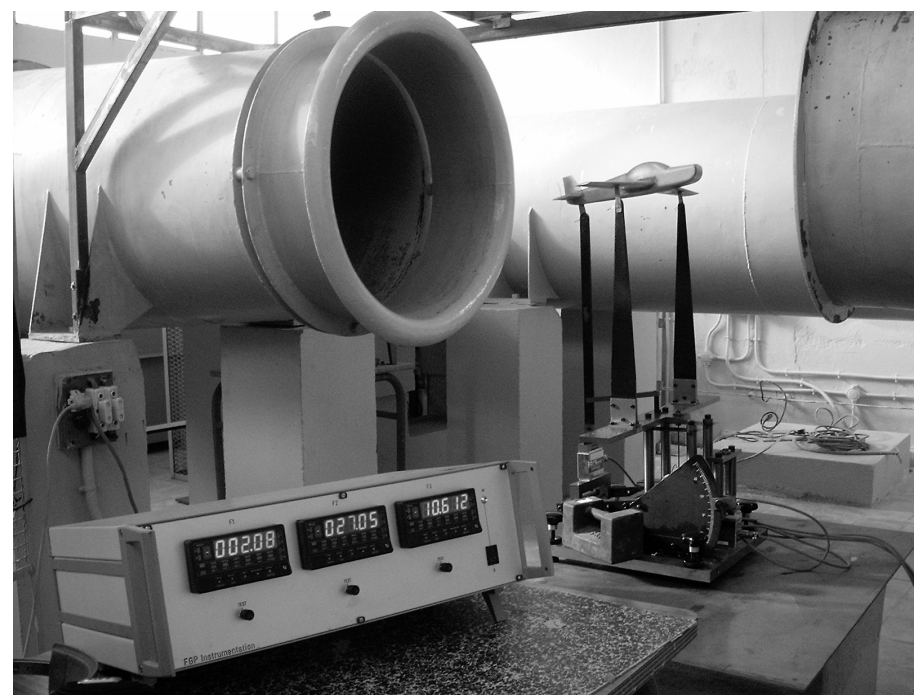

Figure 2: Experimental setup.

\section{Undamaged and damaged states}

Before studying the influence of repairs, tests on the undamaged and damaged model were realized. The appropriate representation of the results and their comparison between, undamaged, damaged and repaired, is to give the fineness curve as function of the angle of incidence instead of the lift versus drag polar where the angle of incidence does not explicitly appear; the fineness being the ratio between the lift and drag coefficients $\left(\mathrm{C}_{\mathrm{L}} / \mathrm{C}_{\mathrm{D}}\right)$.

The obtained results given in Fig. 3 are used as a reference in the following sections. Besides the aerodynamic performance losses [8], the effect of the damage is also characterized by a displacement of the angle of the maximum fineness towards $10^{\circ}$ instead of $8^{\circ}$ for the undamaged case. This displacement is due to the damage hole providing the upper surface with an additional air flow from the lower surface; disturbing consequently the pressure field distribution. This disturbance affects considerably $\mathrm{C}_{\mathrm{L}}$ and $\mathrm{C}_{\mathrm{D}}$.

\section{Repaired states}

The influences of repairs on the aerodynamic performances at the position 1 of the wing are considered through two different repair parameters: the scheme and the thickness.

\subsection{Influence of repair schemes}

Three repair configurations were studied for each damage scenario, namely: full, upper and lower repairs. In this section, we compare the effects of these configurations for the damage located at the position 1 . The thickness of the circular patch used here was $0.2 \mathrm{~mm}$. 


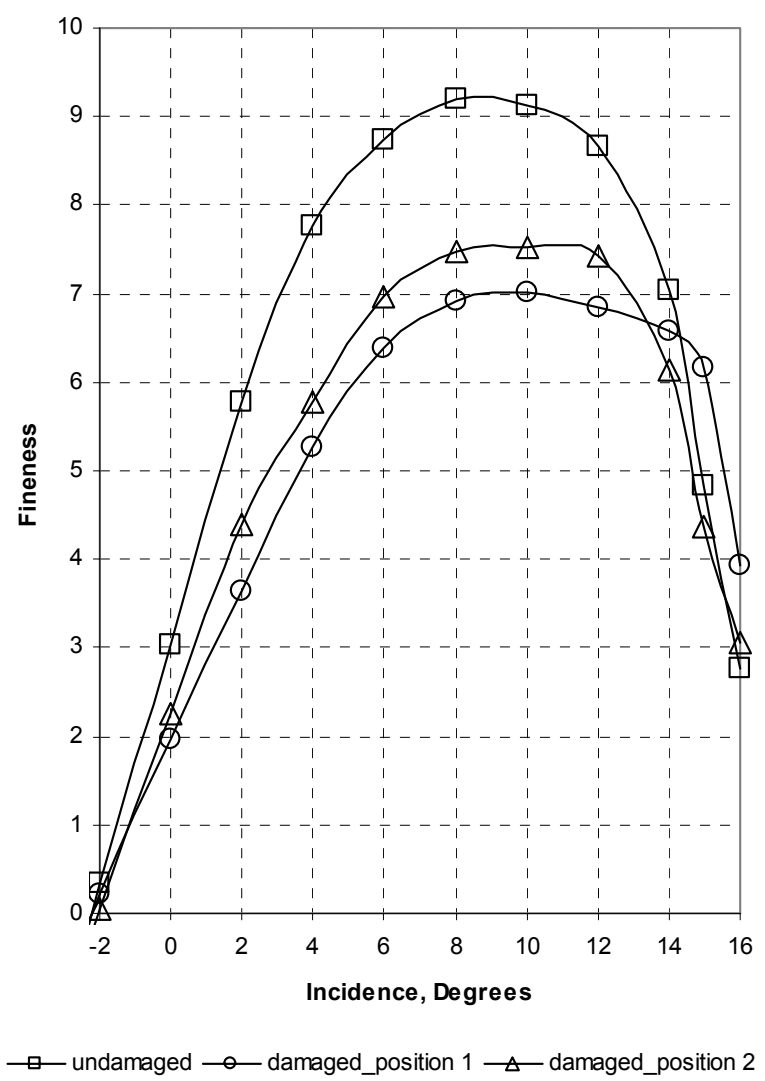

Figure 3: Influence of damage on fineness.

Figure 4 shows that all repairs schemes provided improvements to the fineness values. The full repair, as expected, provides the best improvement over most of the incidence interval; while the upper and lower repair curves are practically the same. It must be outlined as also shown in Fig. 4 that all repairs restore the undamaged angle of maximum fineness value $\left(8^{\circ}\right)$.

For low incidence $\left(\alpha<6^{\circ}\right)$, the fineness values are closer to those of the undamaged state, while above the angle of maximum fineness $\left(\alpha>8^{\circ}\right)$, the improvement (increment) decreases up to $\alpha=14^{\circ}$ (stall angle) [8].

In the case of lower and upper schemes, repairing only one surface (upper or lower) doesn't completely restore the geometry of the wing surfaces so that the upper or lower remained hole acts as a circular cavity, inducing a pressure drag increment. The flow structure around the cavities is a complex study [10] and is beyond the scope of this work. 


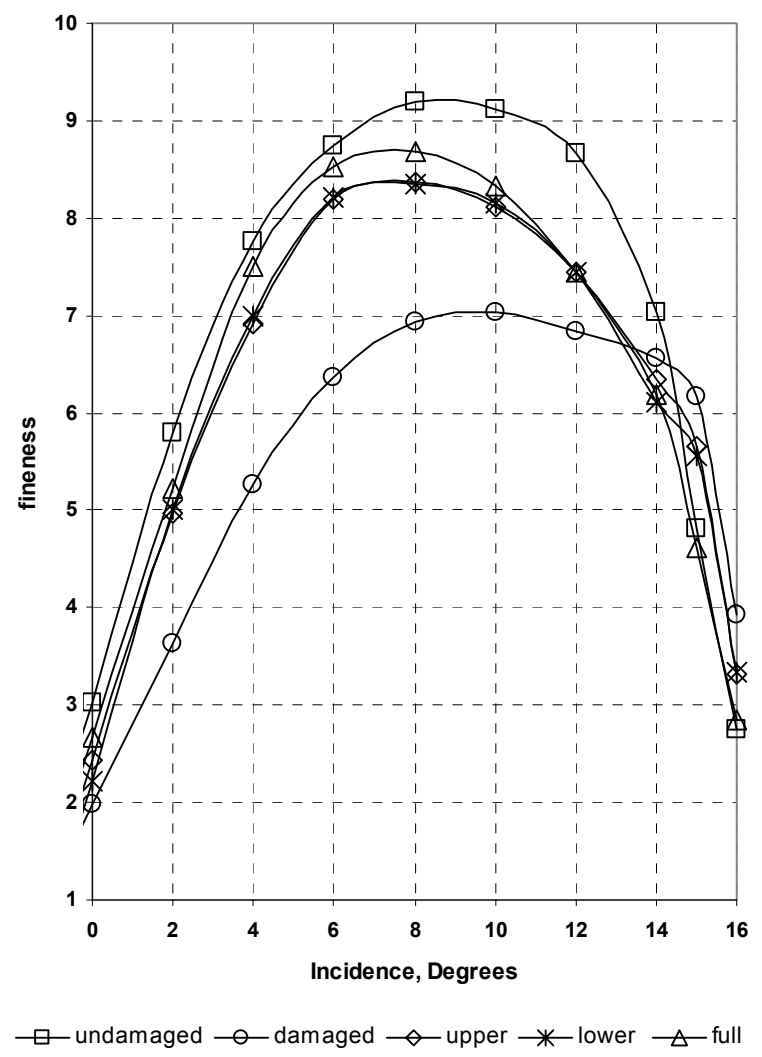

Figure 4: $\quad$ Lift to drag ratio due to repairs.

To appreciate the gain of recovery $\Delta f_{\text {recov }}$ (in percentage) of the fineness from the damaged state, we define, from this state, considered at first as reference, at given incidence and for each type of repairs $\Delta \mathrm{f}_{\text {recov/repairs }}$ as follows:

$$
\Delta f_{\text {recov/repairs }}(\%)=\left(\frac{f_{\text {repair scheme }}-f_{\text {damaged }}}{f_{\text {damaged }}}\right)_{\alpha}
$$

Thus, Fig. 5, obtained from Fig. 4 and Eq. (1), clearly shows:

- All the repair schemes exhibit improvements of the fineness from the damaged state.

- The repair type approaching the undamaged values is the full repair. Thus, we observe that the fineness recovered $\Delta \mathrm{f}_{\text {recov/full repair }}$ varies in this case from $35 \%$ at $0^{\circ}$, up to $45 \%$ at $2^{\circ}$, and then decreases down to $10 \%$ at $12^{\circ}$.

- The upper and lower repairs display the same values, $\Delta \mathrm{f}_{\text {recov/upper repair }}$ and $\Delta \mathrm{f}_{\text {recov/lower repair }}$ for the whole range of incidence, lower than those $\Delta f_{\text {recov/full repair }}$ of the full repair. 


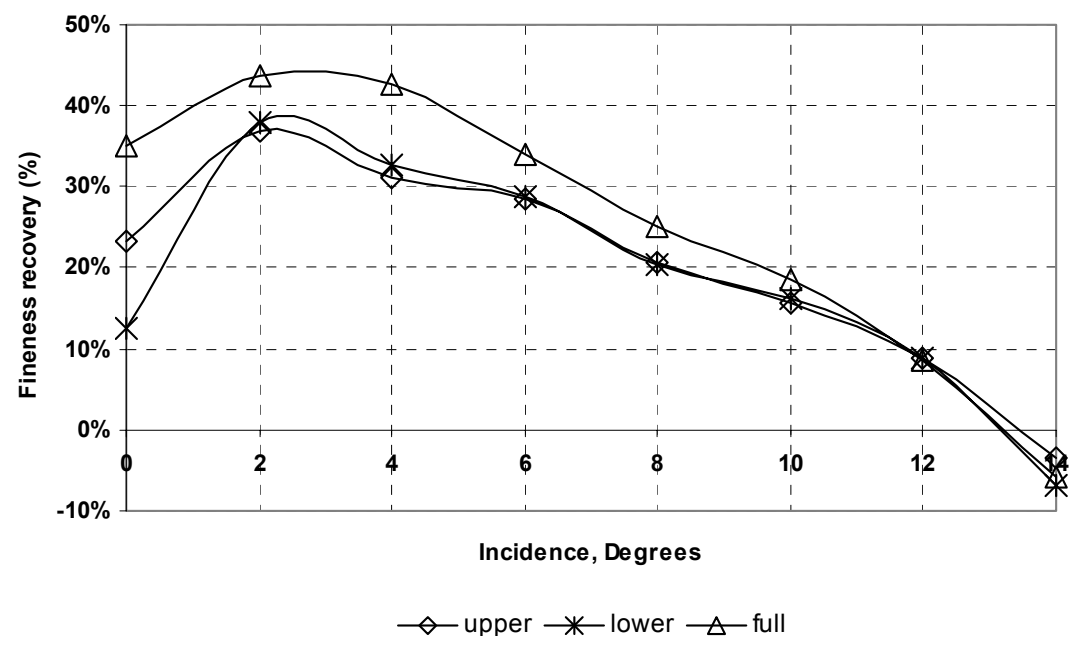

Figure 5: Percentage of recovery in fineness due to repairs.

\subsection{Influence of repair thickness}

In this section, we consider circular patch with a full scheme over the damage hole located at position 1. Measurements were carried out for four plates, of thickness $\mathrm{h}=0.2,0.6,1$ and $1.4 \mathrm{~mm}$. To generalise our results, we consider nondimensional representation of the thickness; the ratio of each value of the used thickness over the value of the local chord. These relative thicknesses will henceforth be referred to as $\overline{\mathrm{e}}_{1}, \overline{\mathrm{e}}_{2}, \overline{\mathrm{e}}_{3}$ and $\overline{\mathrm{e}}_{4}$ equal respectively to $0.3 \%, 1 \%, 1.6 \%$ and $2.3 \%$.

Although some values of the thickness can appear as large regarding the real size of the wing; they are considered in the present study in order to assess the largest acceptable repair thickness value.

It can be noticed in Fig. 6 that all the thicknesses exhibit improvements in the fineness for angle of incidence as large as $14^{\circ}$. Moreover, the fineness improvement decreases with the increase of the relative thickness ( $\overline{\mathrm{e}})$.

For angle of incidence larger than $14^{\circ}$, all the thicknesses used lead this time to fineness value below the fineness of the unrepaired wing.

We can resume that more the values of the thickness are large, less are the angle of incidence values for which the fineness improvement is interesting compared to the unrepaired state. In order to grasp the influence of the thickness on each wing surface (extrados and intrados), we have also consider the lower and the upper repairs where we also find that the variation of the thickness involve different improvements for each scheme repairs. The lower scheme is the less sensitive to the increase of the repair patch for largest value of $\bar{e}$, exhibiting by the way, the best value than the two others even the full scheme (Fig. 7). 


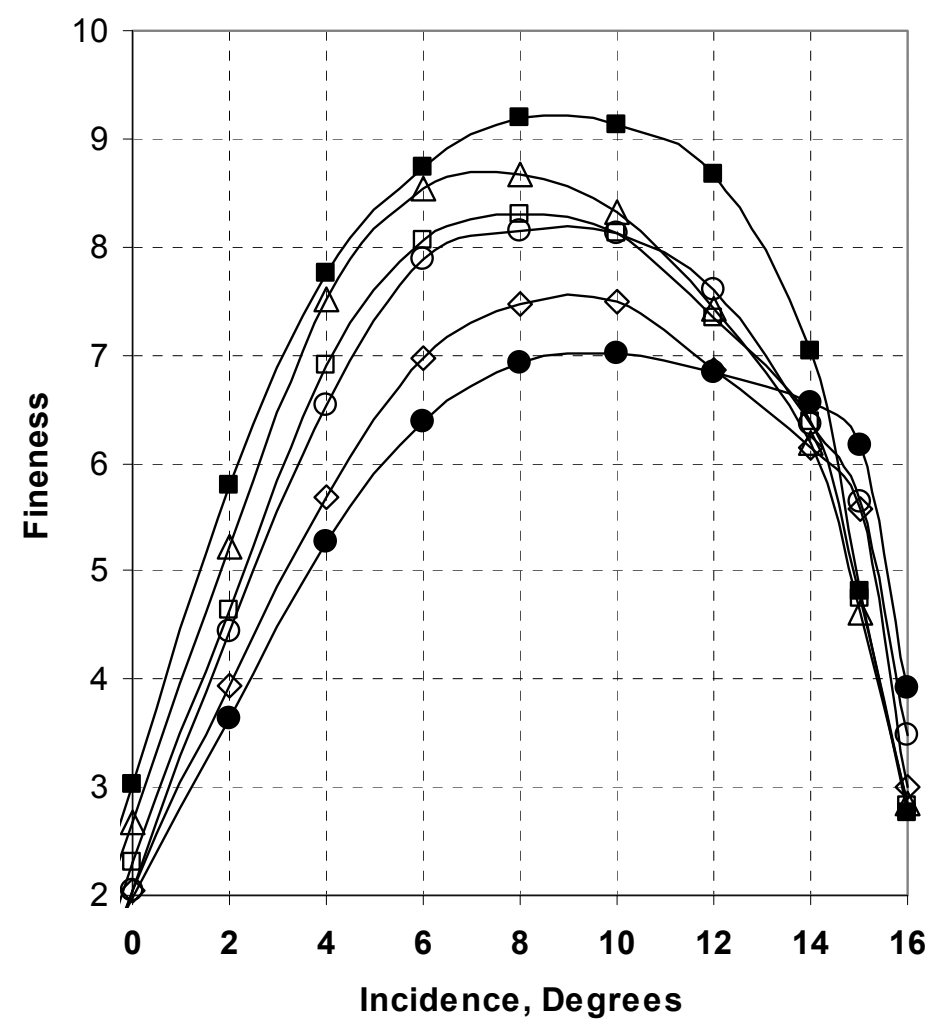

$\triangle \overline{\mathrm{e}} 1 \multimap \overline{\mathrm{e}} 2 \multimap-\overline{\mathrm{e}} 3 \multimap \mathrm{e} 4 \multimap$ damaged $\rightarrow$ undamaged

Figure 6: Influence of repair thickness on the fineness.

It can be concluded that the full scheme gives lower performances than the two other as the thickness of the patch increases. This can be explained by the drag induced by the presence of the patches on both surfaces of the wing, compared to the upper and lower repairs where only one surface is involved in the drag increase.

\section{Conclusion}

The present investigation has been conducted for two aspects of the influence of battle damage repair patches on the aerodynamic performance of an aircraft model: the repair scheme and the thickness of the patch.

First of all, it is shown that, for all the scheme repairs, the battle damage repair patches can achieve substantial reductions in drag coefficient as well as increases in lift coefficient, close to those of the undamaged wing depending on the incidence angle. 

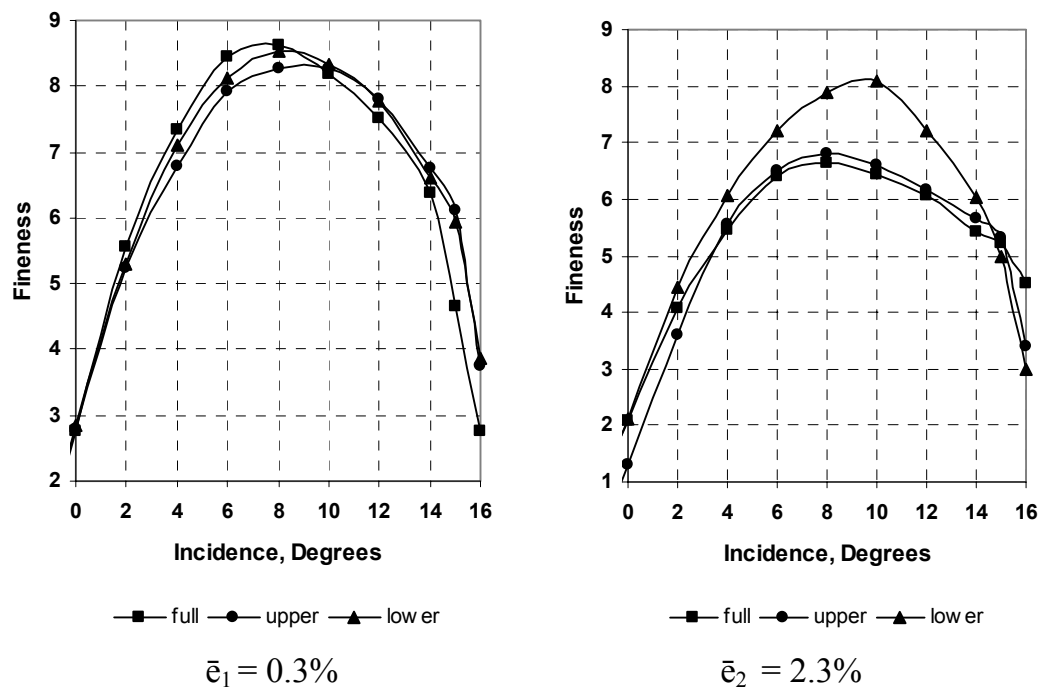

Figure 7: $\quad$ Effect of repair thickness on fineness for different schemes.

Second, the full repair scheme appears, globally, to be the more appropriate to gain aerodynamic performance lost from battle-damage provided that the patch is thin enough.

Repairs to just one surface (upper or lower repairs) also produce significant improvements in lift and drag coefficients. The prevention of flow through the wing seems to be the major factor for the improvement.

Third, the increase of the patch thickness leads to the decrease of the improvement. So that, for a large enough patch thickness, the repair no longer leads to improvement; rather the opposite, relative to the unrepaired (unpatched) model. Consequently, repair by patches showed a compromise between the improvement due to the removal of flow through the damage hole and the performance loss due to the plates installed on the wings to remove the hole.

As a conclusion, to repair the battle-damaged surfaces on the wing, it is preferable to use the full scheme with a circular patch as thin as possible.

\section{References}

[1] Pitt P., Multiple-site and Widespread Fatigue Damage in Aging Aircraft, Engineering Failure Analysis, Vol. 4, December 1997, pp. 237-257.

[2] Jones R., Assessing and Maintaining Continued Airworthiness in the Presence of Wide Spread Fatigue Damage: an Australian Perspective, Engineering Fracture Mechanics, Vol. 60, No. 1, 1998, pp. 109-130.

[3] Jones R., and Chui W. K., Composite Repairs to Cracks in Thick Metallic Components, Composite Structures, Vol. 44, January 1999, pp. 17-29. 
[4] Klug J. C., and Sun C. T., Large Deflection Effects of Cracked Aluminium Plates Repaired with Bonded Composite Patches, Composite Structures, Vol. 42, July 1998, pp. 291-296.

[5] Seo D. C., and Lee J. J., Fatigue Crack Growth Behaviour of Cracked Aluminium Plate Repaired with Composite Patch, Composite Structures, Vol. 57, July 2002, pp. 323-330.

[6] Avdelidis N. P., Moropoulou A., and Marioli Riga Z. P., The Technology of Composite Patches and their Structural Reliability Inspection Using Infrared Imaging, Progress in Aerospace Sciences, Vol. 39, May 2003, pp. 317-328.

[7] Render P. M, De Silva S., Walton A. J., and Mani M., Experimental Investigation into the Aerodynamics of Battle damaged airfoils, Journal of Aircraft, Vol. 44, No. 2, 2007, pp. 539-549.

[8] Djellal S., Ouibrahim A \& Render P. M., The Influence of Battle Damage on the Aerodynamic Characteristics of a Model of an Aircraft. WSEAS Transactions on Fluid Mechanics, Vol.1, January 2006, pp. 89-95.

[9] Djellal S., Ouibrahim A \& Render P. M., Effects of Battle damage repairs on the aerodynamics of a wing, $2^{\text {èmes }}$ Journées Nationales sur l'aéroGazodynamique et Turbomachines, 5-6 December 2005, Oran, Algeria.

[10] Rowley C. W., Juttijudata V., and Williams D. R., Cavity Flow Control Simulations and Experiments, $43^{\text {rd }}$ AIAA Aerospace Sciences Meeting, Reno, NV, January 10-13, 2005. 